\title{
AVALIAÇÃO DO MÉTODO DE SINTONIA IMC COMO ESTRATÉGIA DE CONTROLE PARA UMA PLATAFORMA DE DETECÇÃO DE INCRUSTAÇÃO
}

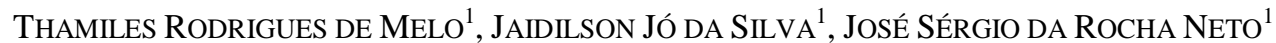

\author{
1. Laboratório de Instrumentação Eletrônica e Controle, Departamento de Engenharia Elétrica, \\ Universidade Federal de Campina Grande \\ Av. Aprígio Veloso, Campina Grande, PB, Brasil \\ E-mails: thamiles.melo@ee.ufcg.edu.br, \\ \{jaidilson, zesergio\} ddee.ufcg.edu.br
}

\begin{abstract}
This article presents the evaluation of IMC method (Internal Model Control) and one your extension applied as a control strategy for dynamic model obtained of the plant in study, in this case, a first order system with time delay which represents the fluidic transport in a didactic platform for fouling detection. In a routine implemented via MATLAB, were obtained for same robustness level, the tuning and performance parameters for the controller project for each strategy, and the simulation results of the close loop control system, given one set-point change and one load disturbance, both of type unit step.
\end{abstract}

Keywords - Fouling Detection, Control Strategies, IMC Method, MATLAB.

Resumo - Neste artigo apresenta-se a avaliação do método IMC (do inglês, Internal Model Control) e uma de suas extensões aplicadas como estratégia de controle para o modelo dinâmico obtido da planta em estudo, neste caso, um sistema de primeira ordem com atraso de transporte que representa o transporte fluídico em uma plataforma didática de detecção de incrustação. Em uma rotina implementada no MATLAB, foram obtidos para um mesmo nível de robustez, os parâmetros de sintonia e de desempenho para o projeto do controlador de cada estratégia, e os resultados de simulações do sistema de controle em malha fechada, dadas uma variação no sinal de referência e uma perturbação de carga, ambas do tipo degrau unitário.

Palavras-chave — Plataforma de Detecção de Incrustação, Estratégias de Controle, Método IMC, MATLAB.

\section{Introdução}

Um grave problema presente em sistemas de transporte fluídico industriais é o lento acúmulo de materiais orgânicos e/ou inorgânicos em suspensão no fluido ao longo da superfície interna do tubo. Este processo de deposição de materiais indesejados, também denotado de processo de formação de incrustação, gera grandes preocupações para o operador, pois com o tempo, essas incrustações vão diminuindo o diâmetro dos tubos (Fig. 1) e tornando necessárias ações periódicas de manutenção, como trocas de tubos e abertura de equipamentos para limpeza (Martin et al, 1996), (Salvador, 2010).

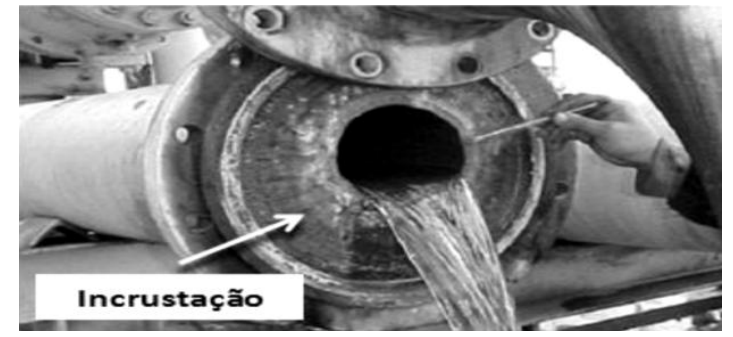

Figura 1. Fotografia de uma tubulação de descarte de água em uma plataforma da Petrobrás com incrustação (Gomes e Costa, 2009).

Devido às graves consequências econômicas acarretadas, a preocupação com incrustações trouxe uma grande quantidade de atividade de pesquisa para as indústrias, procurando-se desenvolver medidas preditivas e preventivas por meio do conhecimento sobre os mecanismos e os fatores associados à in- crustação, tais como variações na vazão, na pressão e na temperatura nos tubos (Mansoori, 2001).

$\mathrm{Na}$ indústria de processos, o controlador PID (Proporcional, Integral e Derivativo) é um dispositivo de controle marcante, com implementações em sistemas embarcados, controladores lógicos programáveis, sistemas de controle distribuído ou em softwares comerciais. Do ponto de vista da simplicidade e eficiência, o controlador PID representa uma solução dinâmica satisfatória no controle de um grande número de aplicações industriais (Knihs et al, 2010).

$\mathrm{O}$ método de sintonia IMC (do inglês, Internal Model Control), onde a precisão do modelo do processo é levada em consideração no controlador, pode ser hibridizado com o controlador PID, pois tem a vantagem de apresentar uma estrutura simples para lei de controle, em que o projeto do controlador está diretamente relacionado a escolha do filtro IMC, tipicamente um filtro passa-baixas com ordem prédefinida. Dessa forma, o parâmetro de ajuste de interesse é a constante de tempo do filtro, que representa um equilíbrio claro entre desempenho em malha fechada e robustez a erros do modelo (Knihs et al, 2010), (Shamsuzzoha e Lee, 2007), (Rivera,1999).

A grande complexidade dos sistemas industriais leva muitos engenheiros a utilizarem ferramentas computacionais de alto desempenho direcionado para o cálculo numérico. Como por exemplo, tem-se o MATLAB (MATrix LABoratory), sistema interativo cujo elemento básico é uma matriz que não requer dimensionamento. Esta ferramenta se tornou uma das mais populares na área de controle, no qual novas versões dos livros didáticos clássicos já incluem 
exercícios para serem resolvidos utilizando esta poderosa ferramenta (Gaspar, Santo e Souza, 2002), (Ibrahim, 2013).

Dessa forma, neste artigo é abordada a avaliação do método IMC e uma de suas extensões aplicadas como estratégia de controle para o modelo dinâmico obtido da planta em estudo, neste caso, um sistema de primeira ordem com atraso de transporte que representa o transporte fluídico em uma plataforma didática de detecção de incrustação. Esta avaliação é realizada utilizando-se uma rotina implementada no MATLAB, no qual pode-se obter, em cada estratégia escolhida, os parâmetros de sintonia e de desempenho para o projeto do controlador e os resultados de simulações do sistema de controle em malha fechada, dadas uma variação no sinal de referência e uma perturbação de carga, ambas do tipo degrau unitário.

\section{Plataforma de Detecção de Incrustação}

Nesta seção apresenta-se as características estruturais do sistema de transporte fluídico em estudo bem como o modelo dinâmico obtido para o mesmo.

\subsection{Características Estruturais}

Tendo em vista um estudo mais aprofundado sobre o processo de incrustação, foi projetada e montada uma plataforma de detecção de incrustação (Fig. 2 ), caracterizada como um sistema de monitoramento distribuído com tubos de aço galvanizado de diferentes diâmetros (1”, 1 1/2”, 2”), cada um com seus sensores de vazão e de pressão, do tipo turbine flowmeters e Strain Gauge, respectivamente. Os tubos de 2" são considerados como tubos principais do sistema, enquanto que os demais são utilizados para geração de distúrbios.

O fluido utilizado no processo, no caso a água, é armazenado em um reservatório de 100 litros e tem sua temperatura medida por meio do sensor LM35 do tipo TO-92 submerso. Há também um módulo de controle da temperatura associado ao par de resistências e a malha de refrigeração interna do reservatório.

Além de válvulas manuais e de uma válvula de controle (do tipo globo, 2 vias, sede simples e diâmetro nominal de 2" com atuador elétrico), há um inversor de frequência que controla a velocidade de rotação do rotor da bomba centrífuga através da variação da frequência de operação adotada.

\subsection{Modelo Matemático da Planta}

Devido a grande maioria dos sistemas reais poderem ser satisfatoriamente representados por modelos de primeira ordem com atraso de transporte, visto a facilidade de explicitar a dinâmica da planta de maneira simplificada, utilizou-se então, da teoria de identificação de sistemas em malha aberta para obter o modelo da planta em estudo.

Com base em testes realizados, utilizando-se a função ident do MATLAB para tratamento dos dados, o modelo dinâmico adotado para a planta foi o representado na Equação (1), já que apresentava maior ganho e menor atraso.

$$
G(s)=\frac{Q(s)}{F(s)}=\frac{K e^{-L s}}{\tau s+1}=\frac{0,60393 e^{-2,5504 s}}{5,4618 s+1}
$$

onde: $G(s)$ é a função de transferência do sistema, no qual representa a relação entre a vazão no tubo de 2" (saída $Q(s)$ ) e a frequência de operação do inversor de frequência (entrada $F(s)$ ); $K$ é o ganho do sistema; $L$ é o atraso de transporte e $\tau$ é a constante de tempo.

O modelo dinâmico de segunda ordem com atraso de transporte também foi levantado, conforme a Equação (2). Porém, tal modelo não foi adotado devido a segunda constante de tempo $\left(\tau_{2}\right)$ ter sido muito pequena com relação à primeira $\left(\tau_{1}\right)$; preferindo-se assim, utilizar apenas o modelo de primeira ordem para o sistema.

$$
G(s)=\frac{K e^{-L s}}{\left(\tau_{1} s+1\right)\left(\tau_{2} s+1\right)}=\frac{0,604 e^{-2,3608 s}}{(5,5358 s+1)(0,1558 s+1)}
$$

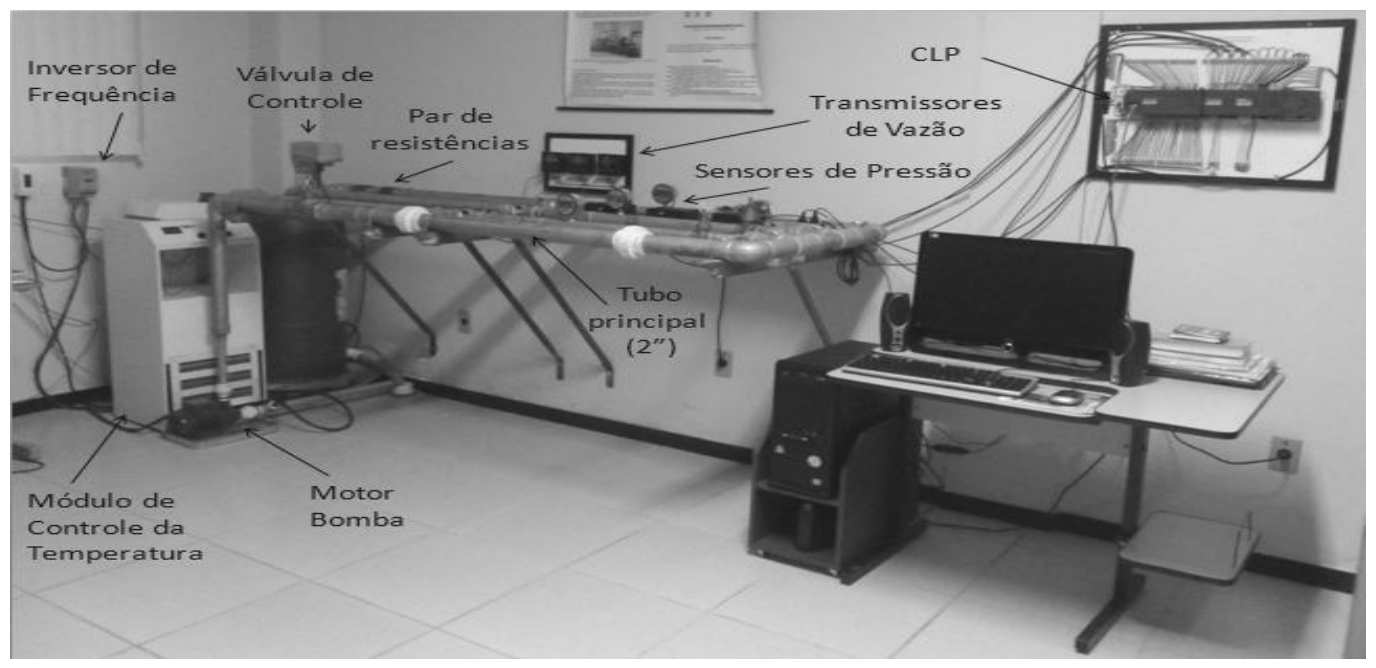

Figura 2. Fotografia da Plataforma de Detecção de Incrustação. 


\section{IMC e Extensões do Método}

Nesta seção propõe-se o método de sintonia IMC e uma de suas extensões como estratégias de controle para o modelo da planta, no caso, a proposta por Shamsuzzoha e Lee (2007).

\subsection{Estratégia IMC}

Neste tipo de método, a principal ideia é conectar o modelo da planta $\tilde{G}_{p}(s)$ em paralelo com a planta real $G_{p}(s)$ e direcionar o controlador $C_{I M C}(s)$ para ter a forma da dinâmica inversa do modelo (Fig. 3) (Knihs et al, 2010). Logo, quanto mais próximo for o modelo com planta real (i. e., $G_{p}(s)=\tilde{G}_{p}(s)$ ), melhor será a robustez do controlador projetado.

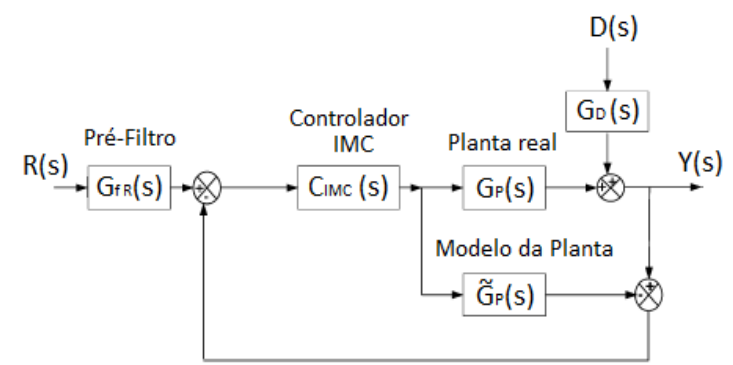

Figura 3. Diagrama de controle da estratégia IMC.

Como $\tilde{G}_{p}(s)$ pode decompor-se em duas partes

$$
\tilde{G}_{p}(s)=P_{\mathrm{M}}(s) P_{\mathrm{A}}(s)
$$

onde: $P_{\mathrm{M}}$ é a porção inversível do modelo (fase mínima) pelo controlador e $P_{\mathrm{A}}$, a porção não- inversível (fase não-mínima que contém o atraso de transporte e/ou zeros no semi-plano direito), então o controlador IMC para sistemas de controle SISO é dado por:

$$
C_{I M C}(s)=P_{\mathrm{M}^{-1}}(s) G_{f}(s)
$$

sendo $G_{f}(s)$ é um filtro passa-baixas da forma:

$$
G_{f}(s)=\frac{1}{(\lambda s+1)^{n}}
$$

onde: $n$ é a ordem do filtro e $\lambda$ é a constante de tempo do filtro, dominante em malha fechada, que visa garantir a implementação do sinal de controle, estabilidade e robustez da malha (Knihs et al, 2010), (Rivera, 1999).

Logo, o controlador equivalente de um sistema com realimentação unitária clássico será dado por

$$
G_{c}(s)=\frac{C_{I M C}(s)}{1-\tilde{G}_{p}(s) C_{I M C}(s)}
$$

onde tem-se que:

$$
\frac{Y(s)}{R(s)}=\frac{G_{c}(s) G_{p}(s) G_{f R}(s)}{1+G_{c}(s) G_{p}(s)}
$$

$$
\frac{Y(s)}{D(s)}=\frac{G_{D}(s)}{1+G_{c}(s) G_{p}(s)}
$$

Tendo-se no sistema um atraso de transporte com aproximação de Padé 1/1, obtém-se a partir de (6) um controlador PID com parâmetros (Rivera, 1999):

$$
\begin{aligned}
G_{c}(s)= & K_{c}\left(1+\frac{1}{T_{i} S}+T_{d} s\right) \\
\text { onde: } \quad & K_{c}=\frac{L+2 \tau}{K(L+\lambda)} \\
& T_{i}=\frac{L}{2}+\tau \\
& T_{d}=\frac{L \tau}{L+2 \tau}
\end{aligned}
$$

\subsection{IMC modificado por Shamsuzzoha e Lee (2007)}

Shamsuzzoha e Lee (2007) propuseram modificações no método IMC de forma que, em malha fechada, o sistema apresentasse também uma melhor resposta quanto a rejeição de distúrbios.

Nesta extensão, é utilizado um grau de liberdade extra denominado $\beta$, que permite fazer o cancelamento do pólo em malha aberta $s=-\frac{1}{\tau}$, determinante na resposta lenta para perturbação de carga (Shamsuzzoha e Lee, 2007).

Dessa forma, para um sistema de primeira ordem com atraso de transporte, e assumindo-se que $G_{p}(s)=G_{D}(s)$, tem-se (Shamsuzzoha e Lee, 2007):

$$
\beta=\tau\left[1-\left(\left(1-\frac{\lambda}{\tau}\right)^{3} e^{-\theta / \tau}\right)^{1 / 2}\right]
$$

e os parâmetros do controlador PID dados por:

$$
K_{c}=\frac{T_{i}}{K(3 \lambda-2 \beta+\theta)}
$$

$$
T_{i}=(\tau+2 \beta)-\frac{\left(3 \lambda^{2}-\frac{\theta^{2}}{2}+2 \beta \theta-\beta^{2}\right)}{(3 \lambda-2 \beta+\theta)}
$$

$$
\begin{aligned}
& T_{d}= \\
& \frac{\left(2 \tau \beta+\beta^{2}\right)-\frac{\left(\lambda^{3}+\frac{\theta^{3}}{6}-\beta \theta^{2}+\beta^{2} \theta\right)}{(3 \lambda-2 \beta+\theta)}}{T_{i}}-\frac{\left(3 \lambda^{2}-\frac{\theta^{2}}{2}+2 \beta \theta-\beta^{2}\right)}{(3 \lambda-2 \beta+\theta)} \text { (16). }
\end{aligned}
$$

\subsection{Cálculo dos parâmetros do controlador PID}

Tendo como base os tópicos anteriores desta seção, implementou-se uma rotina no MATLAB, em que variando-se o valor de $\lambda$ em um intervalo de 0 a $2 L$ (máximo valor adotado para que não fosse gerado $T_{i}$ e $T_{d}$ complexos no IMC modificado), pode-se calcular os valores dos parâmetros $K_{c}, T_{i}$ e $T_{d}$ tanto no método IMC, a partir das Equações (10), (11) e (12), como pela extensão proposta por Shamsuzzoha e Lee (2007), utilizando-se as Equações (14) a (16). 
Calculado tais parâmetros, projetou-se os controladores, conforme a Equação (9), utilizando-se a função $t f$ do Control System Toolbox ${ }^{T M}$.

\section{4 Índices de Desempenho}

Nesta seção aborda-se os principais índices para avaliação do desempenho e robustez dos sistemas de controle em malha fechada com os controladores propostos, segundo Skogestad (2003).

\subsection{Valor de Pico da Função de Sensibilidade $\left(M_{s}\right)$}

O valor de pico $M_{s}$ é definido como o máximo valor absoluto da função de sensibilidade em função da frequência, i.e., $S(j w)=\frac{E(j w)}{R(j w)}$.

Logo:

$$
M_{s}=\max |S(j w)|=\max \left|\frac{1}{1+G_{c}(j w) G_{p}(j w)}\right|
$$

onde valores pequenos são desejados para uma melhor margem de ganho e margem de fase. Tipicamente, impõe-se $M_{S}$ com limite superior igual a 2,0.

\subsection{Sobresinal (Overshoot)}

Sobresinal é uma medida de quanto a resposta excede o valor de referência e/ou distúrbio dada uma variação em degrau.

\subsection{Integral do Erro Absoluto (IAE)}

Para avaliar o desempenho em malha fechada, pode-se considerar um degrau unitário para o sinal de referência e perturbação $(r(t)=d(t)=1)$.

Dessa forma, tem-se que o desempenho da saída pode ser dado pela integral do erro absoluto (IAE), erro este provindo do controlador $(e(t)=y(t)-$ $r(t))$.

Matematicamente, tem-se que:

$$
I A E=\int_{0}^{\infty}|e(t)| d t
$$

onde seja o menor valor possível.

\subsection{Avaliação das Estratégias de Controle}

Para determinação do melhor filtro IMC dentre as estratégias escolhidas por meio do controlador PID equivalente, definiu-se primeiramente $M_{s}$ igual a 1,77 , já que este valor garante, para ambas estratégias, um bom nível de robustez do controlador.

Fixado tal índice, obteve-se valores específicos de $\lambda$ para cada estratégia. Assim, a partir dos controladores respectivos e do modelo da planta em (1), implementou-se os sistemas em malha fechada $\frac{Y(s)}{R(s)}$ e $\frac{Y(s)}{D(s)}$, conforme (7) e (8), a partir da função feedback do mesmo toolbox, considerando $G_{f R}(s)=1$.
Por meio da função step, pode-se simular a resposta de tais sistemas aplicando-se uma variação no sinal de referência e uma perturbação de carga, ambas do tipo degrau unitário.

Pode-se avaliar também os valores resultantes para Overshoot e IAE, conforme descrito em (18) e implementados na rotina do MATLAB.

\section{Resultados e Discussão}

Conforme descrito na seção 3.3, variando-se $\lambda$ em um intervalo de 0 a 5,1 (já que o atraso $L$ da planta é igual a 2,5504), obteve-se os valores de $K_{c}, T_{i}$ e $T_{d}$ para ambas as estratégias, como observado nos gráficos das Figs. 4 a 6.

Quanto a Fig. 4, pode-se notar, para ambas as estratégias, a relação de proporção inversa entre o ganho proporcional $K_{c}$ e a constante de tempo do filtro $\lambda$, devido o comportamento hiperbólico apresentado.

Já nas Fig. 5 e 6, observa-se a invariância paramétrica das constantes de tempo integral $T_{i}$ e derivativa $T_{d}$ no método IMC; enquanto que ao IMC modificado, percebe-se um ajuste dinâmico das ações integral e derivativa na medida que $\lambda$ aumenta.

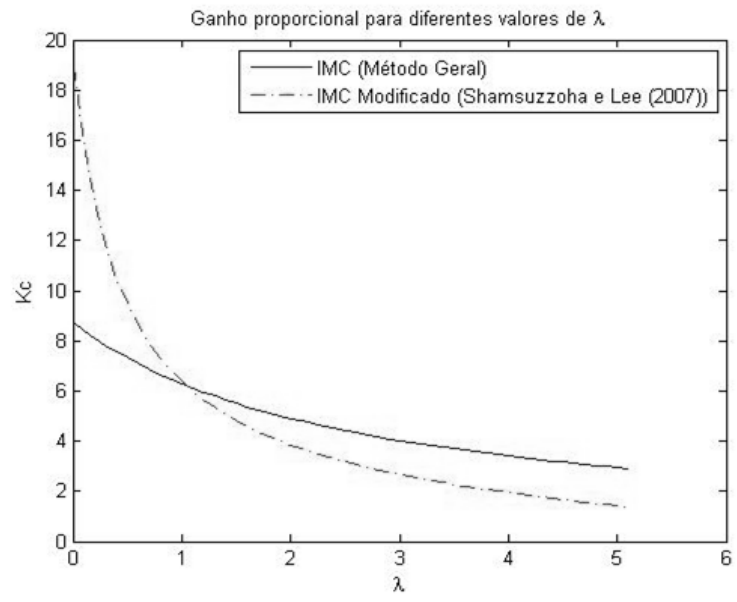

Figura 4. Parâmetro $K_{c}$ para diferentes valores de $\lambda$, em ambas estratégias de controle.

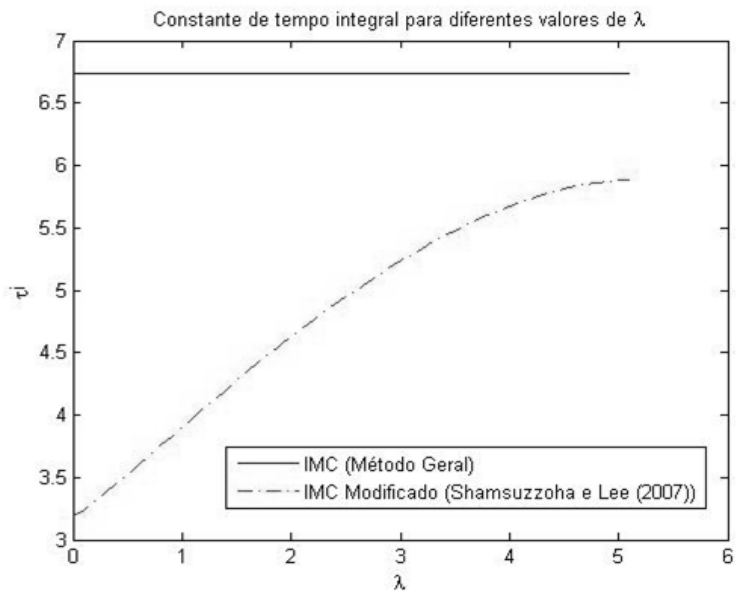

Figura 5. Parâmetro $T_{i}$ para diferentes valores de $\lambda$, em ambas estratégias de controle. 


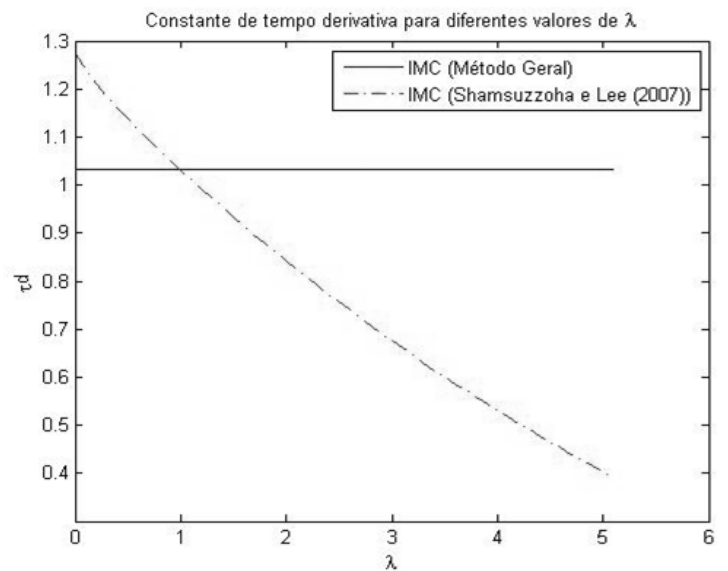

Figura 6. Parâmetro $T_{d}$ para diferentes valores de $\lambda$, em ambas estratégias de controle.

Para avaliação das estratégias de controle propostas, conforme exposto na seção 4.4 , os valores obtidos para constante de tempo do filtro quando $M_{S}$ $=1,77$, foram $\lambda=5,1$ para o método IMC, e $\lambda=2,7$, para o IMC modificado por Shamsuzzoha e Lee (2007).

De posse desses valores, os controladores projetados respectivos foram:

$$
\begin{gathered}
G_{C_{-} I M C}(s)=2,9163\left(1+\frac{1}{6,7370 s}+1,0338 s\right)(19), \\
G_{C_{-} I M C_{-} S L}(s)=2,9582\left(1+\frac{1}{5,0680 s}+0,7235 s\right)(20) .
\end{gathered}
$$

Os sistemas em malha fechada resultantes foram representados em modelo de espaço de estados no MATLAB devido a existência de atraso interno igual a 2,55, proveniente do modelo da planta.

Ao aplicar uma variação no sinal de referência e uma perturbação de carga, ambas do tipo degrau unitário, a resposta destes sistemas foi simulada, conforme os gráficos das Figs. 7 e 8.

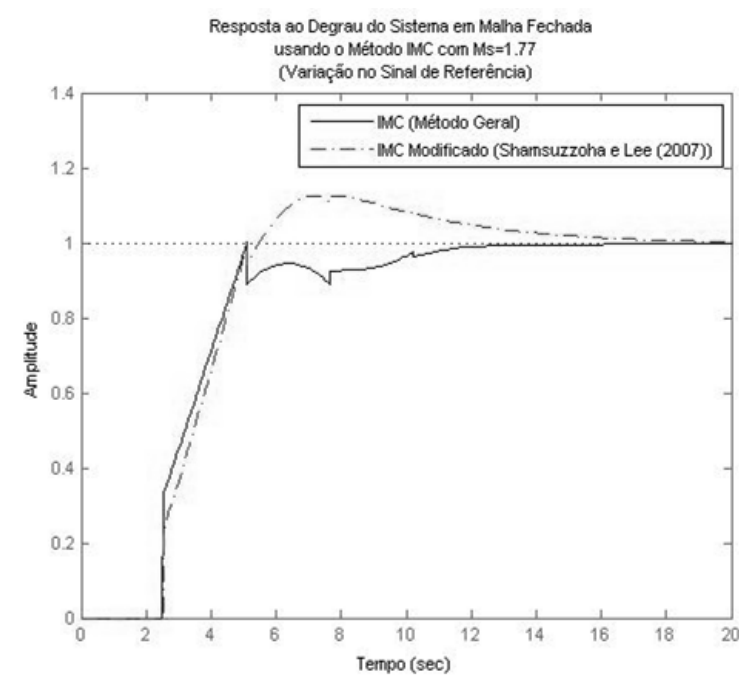

Figura 7. Simulação da resposta do sistema em malha fechada ao degrau unitário, segundo variação no sinal de referência, em ambas estratégias de controle.

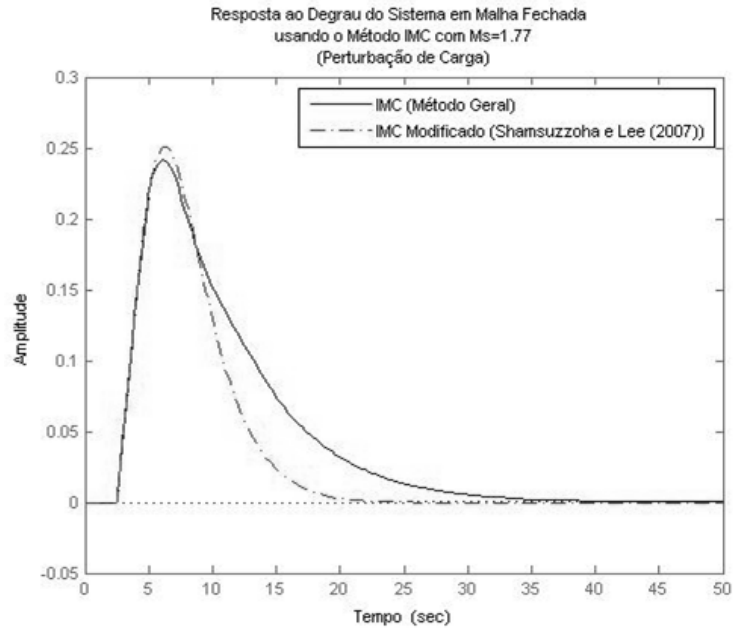

Figura 8. Simulação da resposta do sistema em malha fechada ao degrau unitário, segundo perturbação de carga, em ambas estratégias de controle.

No que diz respeito ao comportamento do sistema em malha fechada na Fig. 7, dada uma variação no sinal de referência, observa-se que a sintonia obtida via IMC é mais agressiva que a do IMC modificado, pois além de ter apresentado uma curva sem overshoot e com formato irregular, minimizou a integral do erro absoluto, alcançando-se um IAE = 3,8146. Diferentemente da outra estratégia, que apresentou uma curva mais suave com overshoot igual a 1,13 e IAE $=4,2633$.

Já quando aplicada uma perturbação de carga, mesmo ambas as estratégias apresentando um overshoot em torno de 0,25 , é perceptível que o IMC modificado obteve um melhor resultado, devido ter alcançado um IAE $=23,5103$, bem menor que da outra estratégia, com IAE $=43,6480$.

\section{Conclusão}

No presente trabalho pode-se realizar a avaliação do método de sintonia IMC e uma de suas extensões, no caso, a proposta por Shamsuzzoha e Lee (2007), como estratégia de controle para atuar no transporte fluídico de uma plataforma didática de detecção de incrustação, cujo modelo dinâmico adotado foi um sistema de primeira ordem com atraso de transporte.

Utilizando-se índices de desempenho como o $M_{s}$, Overshoot e IAE, pode-se comparar quantitativamente o comportamento da planta com cada estratégia definida, dadas uma variação no sinal de referência e uma perturbação de carga, ambas do tipo degrau unitário.

Sendo assim, a melhor estratégia a ser adotada para a planta em estudo é o IMC modificado por Shamsuzzoha e Lee (2007), pois além de apresentar uma boa rejeição a distúrbios, confirmando o que era esperado teoricamente, consegue-se valores de $M_{s}$ bem menores que 1,77 (neste caso, era o melhor valor possível para o método geral). Logo, pode-se alcançar dessa forma um melhor nível de robustez com menor agressividade da sintonia. 


\section{Agradecimentos}

Ao CNPq pelo financiamento do projeto.

\section{Referências Bibliográficas}

Gaspar, P. D.; Santo, A. E.; Souza, J. A. M. F. de. (2002). Apontamentos de MATLAB: Introdução ao MATLAB. Disponível em: <http://webx.ubi.pt/ davide/intro_matlab.pdf>. Acesso em: mar. 2013.

Gomes, R. M., Costa, R. F. D (2009). Garantia de Escoamento: Incrustações Inorgânicas (Apostila). Universidade Petrobrás, Salvador.

Ibrahim, R. C. (2013). MATLAB para o Laboratório de Automação II (verão preliminar). Engenharia Mecatrônica, Escola Politécnica da USP, Universidade de São Paulo, São Paulo.

Knihs et al. (2010). Aplicação da Sintonia IMC no Controlador PID em Sistemas Não-Lineares Simulados com ScicosLab. In: 9th IEEE IAS International Conference Industry Applications, São Paulo, Brazil. Disponível em: $<$ http://ieeexplore.ieee.org/xpl/login.jsp?tp=\&ar number $=5739885 \&$ url=http $\% 3 \mathrm{~A} \% 2 \mathrm{~F} \% 2$ Fieeexplore.ieee.org\%2Fxpls52Fabs_all.jsp\%3Farnumber\%3D5739885>. Acesso em: maio 2013.

Mansoori, G. A (2001). Deposition and Fouling of Heavy Organic Oils and Other Compounds. In: 9th International Conference on Properties and Phase Equilibria for Product and Process Design, Japão. Disponível em: <www.gbhenterprises.com/japanconfpaper.pdf >. Acesso em: jan. 2013.

Martin et al. (1996). Materials Containing Natural Radionuclides in Enhanced Concentrations, EUR 17625, Contract n. ${ }^{\circ}$ B43070/95/00387/MAR/C3.

Rivera, D. E. (1999). Internal Model Control: A Comprehensive View. Disponível em: <http://www.eq.ufrj.br/links/h2cin/eqe709/AUL A_6/RIVERA.pdf>. Acesso em: maio 2013.

Salvador, A. (2010). Noções de Corrosão e Incrustação (Apostila de treinamento - Petrobrás). Disponível em: <http://pt.scribd.com/doc/ 95878654/No-es-de-Corros-o-e-Incrusta-o-1>. Acesso em: dez. 2012.

Shamsuzzoha, M., Lee, M. (2007). IMC-PID Controller Desing for Improved Disturbance Rejection of Time-Delayed Processes. In: Ind. Eng. Chem., Vol. 46, No. 7, pp. 2077-2091. DOI: 10.1021/ie0612360

Skogestad, S (2003). Simple analytic rules for model reduction and PID controller tuning. In: Journal of Process Control 13, pp. 291 - 309. DOI: 10.1016/S0959-1524(02)00062-8 